\title{
ARBORIZAÇÃO DO BAIRRO CENTRO DA CIDADE DE ARACAJU, SERGIPE, E SEUS ORGANISMOS ASSOCIADOS
}

Elaine Cristine do Amarante Matos ${ }^{1}$, José Elvino do Nascimento-Júnior ${ }^{2}$, Dante Luís da Silva Mariano $^{3}$, Aline Lima de Oliveira ${ }^{4}$

(recebido em 03.03.2010 e aceito para publicação em 15.12.2010)

\section{RESUMO}

A urbanização vem se tornando objeto de estudos para ecologistas, já que é um processo que leva à redução dos elementos naturais e à perda de habitats, seja pela destruição total de ecossistemas ou pela substituição parcial da flora nativa por vegetação introduzida. Objetivou-se levantar as espécies arbóreas que estão plantadas no Bairro Centro de Aracaju - SE, e determinar suas origens quanto a exóticas ou nativas ao Brasil e da região nordeste. Além disso, observou-se a ocorrência de organismos habitando ou forrageando nestas árvores. Todas as ruas e praças do bairro centro do município de Aracaju-SE foram visitadas, amostrando-se todas as árvores encontradas e que apresentaram CAP $\geq 15 \mathrm{~cm}$. Foram contabilizadas 1075 árvores nas ruas e praças do bairro Centro, distribuídas em 19 famílias e 65 espécies. As famílias mais representativas foram Arecaceae (15 espécies), Fabaceae (13 espécies) e Bignoniaceae (06 espécies). Formigas, briófitas, epífitas, cupins e ervas-de-passarinhos foram observadas em associação com as árvores. $\mathrm{O}$ bairro Centro apresenta um quantitativo arbóreo significativo, porém com uma riqueza de espécies pequena, o que caracteriza uma arborização homogênea.

PALAVRAS-CHAVE: arborização urbana, espécies exóticas, insetos urbanos.

1. Estudante de graduação em Ciências Biológicas Licenciatura; Universidade Federal de Sergipe, Departamento de Biologia, São Cristóvão - SE. E-mail: elaine.matos@globo.com

2. Licenciado em Ciências Biológicas. Mestrando em Biologia Vegetal. Universidade Estadual de Campinas - UNICAMP. E-mail: jenascimentojr@gmail.com

${ }^{3}$. Licenciado em Ciências Biológicas. Mestrando em Ecologia e biomonitoramento. Universidade Federal da Bahia - UFBA. dante2bio@yahoo.com.br

${ }^{4}$. Licenciado em Ciências Biológicas. Mestrando em Educação. Universidade Federal Rural do Rio de Janeiro-UFRRJ. E-mail: linezinha_blue@hotmail.com

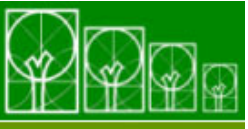

$\mathbf{S} \cdot \mathbf{B} \cdot \mathbf{A} \cdot \mathbf{U}$ Soc. Bras. de Arborização Urbana 


\section{TREE PLANTING OF ARACAJU'S DOWNTOWN, STATE OF SERGIPE (BRAZIL), AND ITS ASSOCIATED ORGANISMS}

\section{ABSTRACT}

Urbanization has become an object of study for ecologists, since it is a process that leads to the decrease of natural elements and habitat loss, either by the complete destruction of ecosystems or partial replacement of the native flora by introduced vegetation. This research aimed to survey the tree species planted in Aracaju's downtown (state of Segipe) and determine its origins as exotic or native to Brazil and the Brazilian Northeast region. Moreover, we observed some organisms inhabiting or feeding themselves in these trees. All streets and squares in Aracaju's downtown were visited and samples of all the trees found were collected, presenting CAP $\geq 15 \mathrm{~cm}$. We identified 1075 trees in the streets and squares of Aracaju's downtown, distributed among 19 families and 65 species. The most representative families were Arecaceae (15 species), Fabaceae (13 species), and Bignoniaceae (6 species). Ants, epiphytes, bryophytes, termites, and mistletoes were observed in association with the trees. Aracaju's downtown presents a significant quantity of trees, however, it has a small variety of species, constituting a homogeneous tree planting.

KEYWORDS: urban tree planting, exotic species, urban insects.

\section{INTRODUÇÃO}

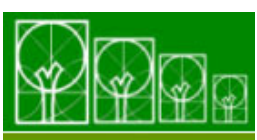


Os ambientes urbanos vêm se tornando objeto de estudos uma vez que grande parte da população mundial já vive em áreas urbanizadas profundamente alteradas pelo estabelecimento humano. Pickett et al. (2001) apresentam duas visões de ecologia urbana: a primeira refere-se a estudos de distribuição e abundância de organismos dentro e ao redor das cidades e seus aspectos biogeoquímicos; a segunda foca em modelar o ambiente para o bem-estar das pessoas e diminuir os impactos das regiões urbanas no ambiente. As cidades têm várias características que não são encontradas no ambiente natural, como a fragmentação dos habitats por prédios e ruas (TANGLEY, 1986). O processo de urbanização de uma área leva à redução dos elementos naturais e a perda de habitats, seja pela destruição total de ecossistemas ou pela substituição parcial da flora nativa por vegetação introduzida. Um dos impactos da urbanização no ecossistema natural é a introdução de espécies exóticas, como ressaltam Dreistadt et al. (1990), Zipperer et al. (2000), Pickett et al. (2001), McKinney (2002) e Tratalos et al. (2007).

As árvores possuem diversas funções importantes em um ambiente urbanizado, influenciando no microclima local, fornecendo sombra, frescor e umidade (OKE et al, 1989); diminuem a velocidade dos ventos e atenuam os efeitos das ilhas de calor (LIRA-FILHO et al., 2001); atuam no controle de erosão, principalmente de encostas íngremes e na proteção das bacias hidrográficas, e enfraquecem a poluição sonora e do ar, contribuindo para o bem-estar físico e social da população (MURPHY, 1997). Além disso, também oferecem boas condições de abrigo e forrageamento para os animais (DAVIS, 1976; FORTUNATO e RUSZCZYK, 1997; WHITMORE et al., 2002), atraindo a avifauna de volta às cidades (LIRAFILHO et al., 2001).

Embora um grande número de estudos sobre árvores urbanas tenha sido realizado em todo o mundo, o conceito de arborização urbana ou florestas urbanas ainda é muito discutido (MAGALHÃES, 2006). Neste estudo, entende-se arborização urbana pelo conjunto das árvores plantadas em praças públicas e na malha viária das cidades. O uso do termo florestas urbanas não é adequado, pois este designa agrupamentos contínuos de árvores que têm relações específicas com os componentes bióticos e abióticos locais.

As cidades oferecem grande variedade de tipos de solo e condições de habitat, podendo dessa forma abrigar várias espécies de formigas e outros artrópodes que são consideradas muitas vezes como pragas urbanas, à medida que potencialmente causam transtornos em residências, fábricas, pomares domésticos, etc. (SOARES et al., 2006). Além disso, as formigas podem transportar agentes patogênicos com facilidade, representando 
dessa forma uma potencial fonte para propagação de infecções hospitalares (COSTA et al., 2006).

O presente estudo tem como objetivo levantar as espécies arbóreas que estão plantadas no Bairro Centro de Aracaju, capital de Sergipe, e determinar suas origens quanto a exóticas ou nativas da região nordeste. Além disso, observar a ocorrência de organismos habitando ou forrageando nestas árvores, diagnosticando quais as espécies arbóreas sustentam maior e menor riqueza de organismos associados.

\section{MATERIAIS E MÉTODOS}

\section{Área de estudo}

A cidade de Aracaju, capital do estado de Sergipe, é popularmente considerada a primeira cidade planejada do Brasil. Contudo, alguns autores (VILAR, 2006; SANTOS e VARGAS, 2007) afirmam que Aracaju é na verdade uma cidade projetada, e não planejada, uma vez que o planejamento urbano leva em consideração a provável situação da cidade em longo prazo, o que não ocorreu neste caso. Na época de sua criação, Aracaju resumiase a um quadrado de aproximadamente $1 \mathrm{~km}$ de cada lado, que corresponde ao atual Bairro Centro, e surgiu através da destruição de dunas, morros e matas e do aterro de rios, canais e manguezais. Atualmente Aracaju possui 544.039 habitantes vivendo em uma área de $181,8 \mathrm{~km}^{2}$ (IBGE, 2009) e sofre com os problemas causados pelo seu crescimento desordenado, entre os quais a destruição de áreas naturais, o surgimento de favelas, problemas de saneamento básico, educação, segurança, saúde e desemprego.

O clima da região é megatérmico sub-úmido úmido, com temperatura média anual de $26{ }^{\circ} \mathrm{C}$. A vegetação natural da cidade era caracterizada por manguezais e restingas, sendo a cidade cortada por duas bacias hidrográficas: a do Rio Sergipe e a do Vaza-Barris (ARAÚJO et al., 2006).

Para o presente estudo foi escolhido o bairro Centro (Fig. 1) da capital do estado de Sergipe, Aracaju. Este foi escolhido por ser uma das áreas que deram início à cidade, representando o modelo de urbanização aqui implantado. Foi projetado pelo engenheiro Sebastião José Basílio Pirro, sendo formado por 32 quadras de $110 \mathrm{~m}^{2}$ cada, com estrutura viária ortogonal (VILAR et al., 2006). 


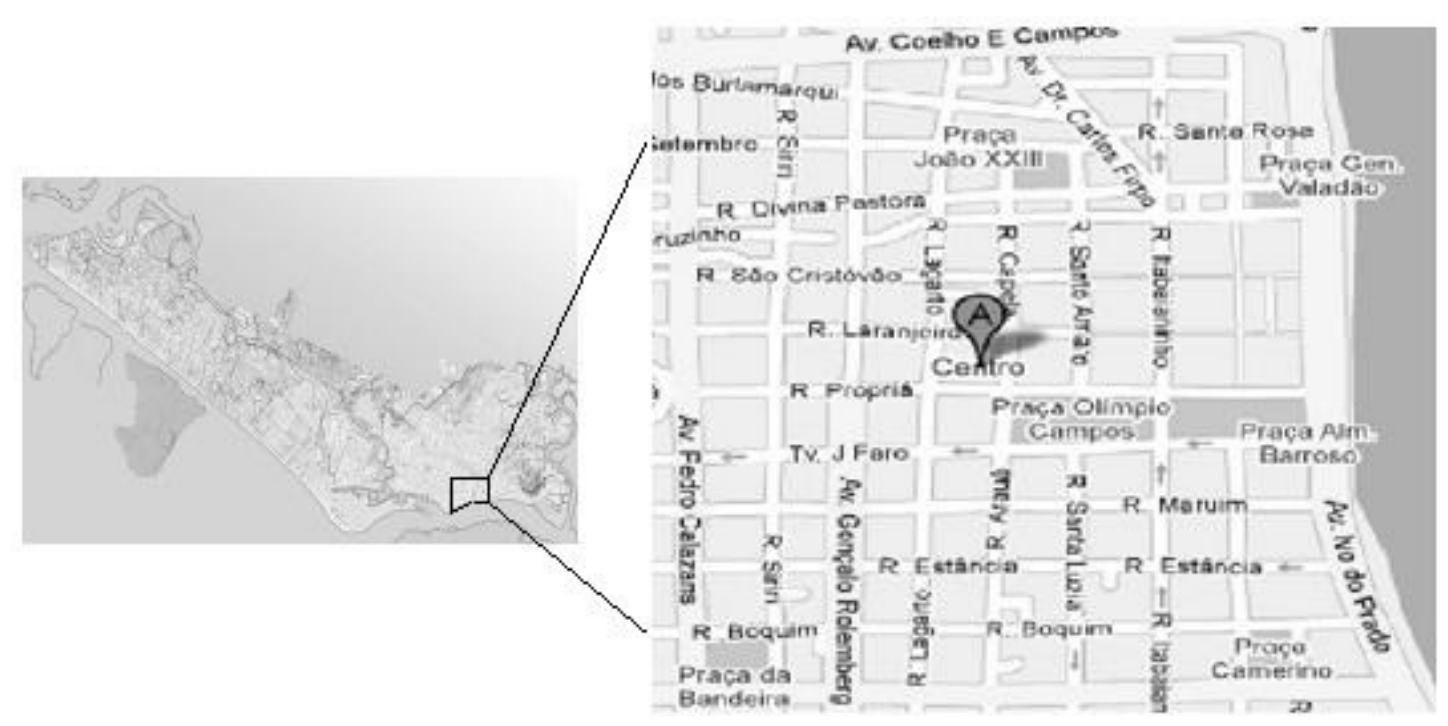

FIGURA 1. Mapa do Bairro Centro de Aracaju, SE. Fonte: GoogleMaps.

FIGURE 1. Map of the District Aracaju's downtown, SE. Source: GoogleMaps.

\section{Coleta e análise dos dados}

Para a realização deste levantamento, todas as ruas e praças do Bairro Centro foram visitadas e percorridas durante os meses de março, abril e maio do ano de 2007 e fevereiro de 2008, totalizando aproximadamente $25 \mathrm{~km}$ de malha viária. Nos logradouros limítrofes do Bairro Centro, apenas os lados voltados para o bairro foram objeto de amostragem. Todas as árvores e arbustos que apresentaram CAP $\geq 15 \mathrm{~cm}$ foram incluídos no estudo. Utilizamos esta medida para evitar a amostragem de plantas jovens ainda não plenamente estabelecidas. Nos casos de plantas que apresentaram múltiplos caules foram incluídas apenas aquelas que possuíam ao menos um caule com CAP $\geq 15 \mathrm{~cm}$. Além disso, observamos a existência de organismos vivos macroscópicos (plantas, fungos e animais) nas plantas amostradas.

As espécies arbóreas foram coletadas de acordo com Fidalgo e Bononi (1984) e identificadas com o auxilio de literatura especializada (LORENZI, 2002; LORENZI et al., 2003) e por comparação com as coleções do herbário ASE (acrônimo segundo HOLMGREEN e HOLMGREEN, 1998), sendo as exsicatas resultantes depositadas no mesmo herbário. O posicionamento das espécies nas famílias botânicas estão conforme APG III (2009). Espécimes da família Arecaceae não foram coletados devido à altura elevada das plantas encontradas e a ausência de material fértil nas mesmas, além das dificuldades apresentadas pelas técnicas de coleta específicas para a família; estas plantas foram identificadas, quando possível, através de literatura especializada (LORENZI et al., 1996). Informações sobre os locais de ocorrência das espécies identificadas foram obtidas 
através de literatura especializada e pela consulta à base de dados SpeciesLink (CRIA, 2008) e ao Banco de Dados de Plantas do Nordeste (CNIP, 2009). Os nomes atualmente aceitos para as espécies foram consultados na base de dados World Checklist of Selected Plant Families (2010), e para aquelas famílias que ainda não receberam tratamento do WCSPF consultamos a base de dados Trópicos (2010).

Como medida da heterogeneidade florística das praças, ruas e avenidas foi usada a equabilidade (PIELOU, 1966), sendo que todas as ruas e avenidas do bairro Centro foram consideradas como uma única unidade amostral. A equabilidade (calculada por J' $=\mathrm{H}^{\prime} / \mathrm{H}_{\max }$, onde $H^{\prime}$ = índice de diversidade de Shannon, $H_{\max }=\operatorname{Ln}(\mathrm{S})$, e $\mathrm{S}=$ número de espécies amostradas) mostra o quanto as abundâncias das espécies encontradas são similares. Neste estudo, uma equabilidade muito próxima a 1 indicaria que as espécies plantadas no Bairro Centro possuem abundância muito similar. Um valor de equabilidade próximo a 0 mostraria matematicamente que algumas espécies possuem sua abundância muito maior que outras em uma dada área.

Morcegos e aves noturnas não foram objeto de amostragem, uma vez que a área não oferece segurança à noite. Alguns exemplares de artrópodes encontrados nas bases ou órgãos aéreos das árvores foram coletados através de busca ativa, e posteriormente identificados no Laboratório de Entomologia da Universidade Federal de Sergipe.

\section{RESULTADOS E DISCUSSÃO}

Grande parte das árvores do Centro de Aracaju sofreram podas drásticas, as quais diminuem suas funções estéticas e de atenuação do microclima local. Além disso, constatamos que as árvores sofrem constantes atos de vandalismos, como cortes profundos em suas cascas e quebra de galhos por moradores. No entanto, os principais danos sofridos pelas árvores são encontrados nas praças e ruas que recebem ornamentação em ocasiões festivas da cidade, como as festas de fim de ano e carnaval. Nestes locais, as árvores servem de suporte para a ornamentação, a qual é fixada por pregos que comumente permanecem nas árvores após a retirada dos enfeites. Os danos que as árvores recebem podem se tornar um meio para entrada de microorganismos causadores de doenças nestas plantas (AUER et al., 2004; FERREIRA e MILANI, 2004).

Após a conclusão do levantamento, foram contabilizadas 1075 árvores nas ruas e praças do bairro Centro, distribuídas em 19 famílias e 65 espécies (Tabela 1), sendo que 44

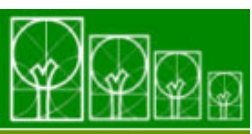

$\mathbf{S} \cdot \mathbf{B} \cdot \mathbf{A} \cdot \mathbf{U}$ Soc. Bras. de Arborização Urbana 
foram identificadas a nível específico, seis a gênero, nove a nível de família e seis espécies permaneceram indeterminadas, uma vez que não entraram em estado reprodutivo durante 0 período do estudo. As famílias mais representativas foram Arecaceae (15 espécies), Fabaceae (13 espécies) e Bignoniaceae (06 espécies).

TABELA 1. Lista das espécies do Bairro Centro, Aracaju (SE), com abundâncias relativa e absoluta e origem geográfica.

TABLE 1. List of species of Aracaju's downtown (SE), with absolute and relative abundances and geographic origin.

\section{Família/Espécie}

$\begin{array}{ccc}\begin{array}{c}\text { Abundância } \\ \text { relativa }\end{array} & \begin{array}{c}\text { Abundância } \\ \text { absoluta }\end{array} & \text { Origem }\end{array}$

\section{ANACARDIACEAE}

Anacardium occidentale L.

$$
0,43 \%
$$

5

Nativa

Mangifera indica L.

$1,40 \%$

15

Exótica

Schinus terebinthifolia Raddi

$0,43 \%$

5

Nativa

Spondias sp.

$0,10 \%$

1

\section{ANNONACEAE}

Annona squamosa $\mathrm{L}$.

$0,10 \%$

1

Nativa

Annona sp.

$0,10 \%$

1

APOCYNACEAE

Cascabela thevetia (L.) Lippold

$\begin{array}{lll}0,30 \% & 4 & \text { Exótica } \\ 0,10 \% & 1 & \text { Exótica } \\ 0,20 \% & 2 & \text { Exótica }\end{array}$

Nerium oleander L.

Plumeria pudica Jacq.

$0,10 \% \quad 1 \quad$ Exótica

Schefflera actinophylla (Endl.) Harms

$\begin{array}{ccc}0,20 \% & 2 & \text { Exótica } \\ 2,50 \% & 27 & \text { Nativa }\end{array}$
Dransf.

\section{ARECACEAE}

Caryota urens L.

13

Exótica

Elaeis oleifera (Kunth) Cortés

$1,20 \%$

Exótica

Leopoldinia piassaba Wallace

$0,43 \%$

5

Nativa

Roystonea oleracea (Jacq.) O.F. Cook

$0,10 \%$

1

Nativa

Thrinax parviflora Sw.

$7,22 \%$

77

Exótica

$0,20 \%$

2

Exótica 


\begin{tabular}{|c|c|c|c|}
\hline Syagrus sp. & $0,10 \%$ & 1 & - \\
\hline Arecaceae sp. 1 & $0,20 \%$ & 2 & - \\
\hline Arecaceae sp. 2 & $0,10 \%$ & 1 & - \\
\hline Arecaceae sp. 3 & $0,10 \%$ & 1 & - \\
\hline Arecaceae sp. 4 & $0,10 \%$ & 1 & - \\
\hline Arecaceae sp. 5 & $0,65 \%$ & 7 & - \\
\hline Arecaceae sp. 6 & $0,10 \%$ & 1 & - \\
\hline Arecaceae sp. 7 & $0,10 \%$ & 1 & - \\
\hline \multicolumn{4}{|l|}{ AURACARIACEAE } \\
\hline Araucaria columnaris (G.Forst.) Hook & $0,10 \%$ & 1 & Exótica \\
\hline \multicolumn{4}{|l|}{ BIGNONIACEAE } \\
\hline $\begin{array}{l}\text { Handroanthus chrysotrichus (Mart. ex A. DC.) } \\
\text { Mattos }\end{array}$ & $0,20 \%$ & 2 & Nativa \\
\hline $\begin{array}{l}\text { Handroanthus impetiginosus (Mart. ex DC.) } \\
\text { Mattos }\end{array}$ & $1,30 \%$ & 14 & Nativa \\
\hline Spathodea campanulata P. Beauv. & $0,30 \%$ & 4 & Exótica \\
\hline Tabebuia sp. & $0,10 \%$ & 1 & - \\
\hline Tecoma stans (L.) Juss. ex Kunth & $0,43 \%$ & 5 & Exótica \\
\hline Bignoniaceae sp. & $0,10 \%$ & 1 & - \\
\hline \multicolumn{4}{|l|}{ CHRYSOBALANACEAE } \\
\hline Licania tomentosa (Benth.) Fritsch & $23,16 \%$ & 249 & Nativa \\
\hline \multicolumn{4}{|l|}{ COMBRETACEAE } \\
\hline Terminalia catappa L. & $1,90 \%$ & 20 & Exótica \\
\hline \multicolumn{4}{|l|}{ CUPRESSACEAE } \\
\hline Cupressaceae sp. & $0,10 \%$ & 1 & - \\
\hline \multicolumn{4}{|l|}{ FABACEAE } \\
\hline Adenanthera pavonina L. & $1 \%$ & 11 & Exótica \\
\hline Anadenanthera colubrina (Vell.) Brenan & $0,10 \%$ & 1 & Nativa \\
\hline Andira fraxinifolia Benth. & $0,30 \%$ & 4 & Nativa \\
\hline Caesalpinia echinata Lam. & $0,52 \%$ & 6 & Nativa \\
\hline Calliandra sp. & $0,20 \%$ & 2 & - \\
\hline Clitoria fairchildiana R.A. Howard & $4,41 \%$ & 47 & Nativa \\
\hline Delonix regia (Bojer ex Hook.) Raf. & $0,43 \%$ & 5 & Exótica \\
\hline Leucaena sp. & $0,10 \%$ & 1 & Exótica \\
\hline
\end{tabular}




\begin{tabular}{|c|c|c|c|}
\hline Pithecellobium dulce (Roxb.) Benth. & $21,26 \%$ & 228 & Exótica \\
\hline Prosopis juliflora (Sw.) DC. & $1,30 \%$ & 14 & Exótica \\
\hline Senna siamea (Lam.) H.S. Irwin \& Barneby & $0,80 \%$ & 9 & Exótica \\
\hline Tamarindus indica L. & $5,21 \%$ & 56 & Exótica \\
\hline Fabaceae sp. & $0,52 \%$ & 6 & - \\
\hline \multicolumn{4}{|l|}{ MALVACEAE } \\
\hline Pachira aquatica Aubl. & $1,40 \%$ & 15 & Nativa \\
\hline Thespesia populnea (L.) Sol. ex Corrêa & $0,10 \%$ & 1 & Exótica \\
\hline \multicolumn{4}{|l|}{ MELIACEAE } \\
\hline Azadirachta indica A. Juss. & $0,20 \%$ & 2 & Exótica \\
\hline Cedrela fissilis Vell. & $0,20 \%$ & 2 & Nativa \\
\hline \multicolumn{4}{|l|}{ MORACEAE } \\
\hline Ficus benjamina L. & $8,52 \%$ & 91 & Exótica \\
\hline Ficus microcarpa L. f. & $4,30 \%$ & 46 & Exótica \\
\hline \multicolumn{4}{|l|}{ MYRTACEAE } \\
\hline Syzygium cumini (L.) Skeels & $0,20 \%$ & 2 & Exótica \\
\hline Syzygium malaccense (L.) Merr. \& L.M. Perry & $0,20 \%$ & 2 & Exótica \\
\hline \multicolumn{4}{|l|}{ POLYGONACEAE } \\
\hline Triplaris caracasana Cham. & $1 \%$ & 11 & Exótica \\
\hline \multicolumn{4}{|l|}{ RUSCACEAE } \\
\hline Dracaena fragrans (L.) Ker Gawl. & $2,30 \%$ & 25 & Exótica \\
\hline \multicolumn{4}{|l|}{ SAPINDACEAE } \\
\hline Sapindus saponaria L. & $0,10 \%$ & 1 & Nativa \\
\hline \multicolumn{4}{|l|}{ URTICACEAE } \\
\hline Cecropia pachystachya Trécul & $0,10 \%$ & 1 & Nativa \\
\hline \multicolumn{4}{|l|}{ NÃO IDENTIFICADAS } \\
\hline NI 1 & $0,20 \%$ & 2 & 一 \\
\hline $\mathrm{NI} 2$ & $0,10 \%$ & 1 & - \\
\hline NI 3 & $0,27 \%$ & 3 & - \\
\hline $\mathrm{NI} 4$ & $0,10 \%$ & 1 & - \\
\hline NI 5 & $0,20 \%$ & 2 & - \\
\hline $\mathrm{NI} 6$ & $0,10 \%$ & 1 & - \\
\hline TOTAL & $100 \%$ & 1075 & \\
\hline
\end{tabular}


O número de espécies pode ser considerado alto quando comparado a outras cidades brasileiras e ao esforço amostral destes trabalhos (Tabela 2).

TABELA 2. Número de espécies na arborização de algumas cidades brasileiras.

TABLE 2. Number of species in some Brazilian cities tree planting.

\begin{tabular}{|c|c|c|c|c|}
\hline Cidade (Estado) & $\begin{array}{l}\text { Esforço } \\
\text { amostral }\end{array}$ & $\begin{array}{c}\text { № de } \\
\text { espécies }\end{array}$ & $\begin{array}{l}\text { Espécie mais } \\
\text { abundantes }\end{array}$ & Referência \\
\hline $\begin{array}{c}\text { Campina Grande } \\
\text { (PB) }\end{array}$ & Dois bairros & 23 & Ficus sp. & $\begin{array}{c}\text { Meneses et al., } \\
2003\end{array}$ \\
\hline $\begin{array}{l}\text { Campina Grande } \\
\qquad(\mathrm{PB})\end{array}$ & $\begin{array}{l}\text { Toda a } \\
\text { cidade }\end{array}$ & 132 & Prosopis Juliflora & Dantas et al., 2004 \\
\hline Nova Iguaçu (RJ) & Um bairro & 46 & Ficus benjamina & Rocha et al., 2004 \\
\hline Nova Iguaçu (RJ) & Um bairro & 59 & Cassia siamea & Rocha et al., 2004 \\
\hline Assis (SP) & $\begin{array}{l}\text { Toda a } \\
\text { cidade }\end{array}$ & 54 & Licania tomentosa & $\begin{array}{l}\text { Rossato et al., } \\
2008\end{array}$ \\
\hline Goiandira (GO) & $\begin{array}{l}\text { Toda a } \\
\text { cidade }\end{array}$ & 105 & Licania tomentosa & Pires et al., 2007 \\
\hline
\end{tabular}

A maior parte das árvores do Centro de Aracaju pertencem a um pequeno grupo de espécies, sendo as mais abundantes Licania tomentosa (Benth.) Fritsch, da família Chrysobalanaceae, correspondendo a $23,16 \%$ das árvores encontradas, e Pithecellobium dulce (Roxb.) Benth., família Fabaceae, com 21,26\%. A homogeneidade da arborização de Aracaju também foi constatada por Neto et al. (2007) quando analisaram a arborização de algumas das principais avenidas da cidade. $O$ fato da maior parte das árvores do Centro ser de apenas quatro espécies não é recomendado, uma vez que esta condição torna a arborização urbana esteticamente monótona e mais frágil ao ataque de patógenos. Para que isso seja evitado, recomenda-se que uma mesma espécie não corresponda a mais do que 10 a $15 \%$ das árvores de uma cidade (PIVETTA e SILVA FILHO, 2002).

A equabilidade é uma medida simples que pode ser usada para demonstrar como cada espécie está representada em um local em termos de número de indivíduos. Quanto mais elevada a equabilidade, mais similar é o número de indivíduos de cada espécie no local. Desta forma, a baixa equabilidade encontrada na Praça da Bandeira $(0,58)$ deve-se a predominância da espécie $P$. dulce, que corresponde a $55 \%$ das árvores encontradas no 
local, da mesma forma que na Praça Camerino (equabilidade $=0,61$ ), na qual a mesma espécie responde por $32 \%$ das árvores plantadas.

TABELA 3. Equabilidade e quantidade de árvores e espécies dos logradouros do Bairro Centro de Aracaju, SE.

TABLE 3. Evenness and quantity of tree species and public parks of Aracaju's downtown, SE.

\begin{tabular}{lccc}
\hline Logradouro & Equabilidade & № de árvores & $\begin{array}{c}\text { № de } \\
\text { espécies }\end{array}$ \\
\hline Praça General Valadão & 0,83 & 11 & 3 \\
Praça Fausto Cardoso & 0,81 & 112 & 18 \\
Ruas e avenidas & 0,74 & 390 & 27 \\
Praça Olímpio Campos & 0,71 & 276 & 43 \\
Praça Camerino & 0,69 & 157 & 21 \\
Praça da Bandeira & 0,58 & 121 & 20 \\
\hline
\end{tabular}

A heterogeneidade mais elevada da Praça General Valadão $(0,89)$ deve-se a baixa quantidade de espécies no local e a suas abundâncias semelhantes, uma vez que trata-se de uma pequena praça do bairro.

A maior parte dos indivíduos é exótica (em relação ao Brasil), compondo cerca de 64 $\%$ das árvores do Centro, enquanto que $33 \%$ pertencem a espécies nativas (principalmente L. tomentosa) e $3 \%$ das árvores não tiveram área de ocorrência original determinada. Baseando-se nos dados da base SpeciesLink (CRIA, 2008) e do Checklist das Plantas do Nordeste Brasileiro (CNIP, 2009), entre as espécies identificadas apenas 12 são nativas da região Nordeste do Brasil, sendo as demais artificialmente introduzidas na região. Esses dados apontam para uma intensa desvalorização da flora local e para a preferência demasiada por componentes exógenos a vida cotidiana da comunidade.

Entre os organismos associados às árvores destacaram-se as formigas (família Formicidae), com nove morfoespécies, sendo que quatro foram identificadas a nível específico e cinco a nível de gênero (Tabela 4). As espécies Paratrechina longicornis (Latreille) e Tapinoma melanocephalum (Fabricius) (ambas exóticas) estão entre as principais formigas consideradas pragas nas áreas urbanas de diversas regiões brasileiras (CAMPOS-FARINHA et al., 2002).

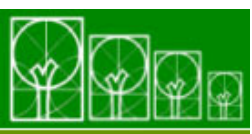

$\mathbf{S} \cdot \mathbf{B} \cdot \mathbf{A} \cdot \mathbf{U}$ Soc. Bras. de Arborização Urbana 
TABELA 4: Espécies de formigas associadas às árvores do Bairro Centro

TABLE 4. Ant species associated with trees in Aracaju's downtown

\section{Espécie}

Camponotus blandus (Smith)

Cephalotes sp. 1

Cephalotes sp. 2

Paratrechina longicornis (Latreille)

Pheidole fallax Mayr

Pseudomyrmex sp.

Tapinoma melanocephalum (Fabricius)

Wasmannia sp. 1

Wasmannia sp. 2

As formigas são bastante comuns nas árvores do Bairro Centro, sendo encontradas em pelo menos $65 \%$ das árvores da área, e geralmente duas ou três espécies eram observadas na mesma árvore, indicando a possibilidade de os recursos disponíveis serem suficientes para todas as espécies. As espécies arbóreas nas quais foram encontradas mais formigas em seus ramos ou bases são L. tomentosa (82\%), P. dulce (80 \%), C. fairchildiana (69 \%) e $T$. indica (62 \%), e estas portanto não são recomendadas para o plantio em áreas próximas a clínicas, maternidades e hospitais, uma vez que potencialmente abrigariam formigas que poderiam posteriormente invadir estes locais, acentuando o risco de dispersão de microrganismos patogênicos.

Entre os outros organismos associados às árvores do bairro centro destacam-se os musgos (plantas avasculares pertencentes ao filo Bryophyta), presentes em 40,75\% das árvores, e as briófitas hepáticas (plantas avasculares pertencentes ao filo Marchantiophyta), que ocorrem em cerca de $58 \%$ das árvores do bairro.

Além das briófitas sensu lato, outras plantas foram encontradas associadas à arborização do Centro em duas condições distintas: como epífitas e como hemiparasitas. As epífitas estão presentes em $12 \%$ das árvores, especialmente sobre as espécies $L$. tomentosa e $P$. dulce, nas quais $71 \%$ das epífitas foram encontradas. A alta incidência de epífitas sobre essas espécies pode ser explicada pela rugosidade de suas cascas, que facilitam a fixação das sementes e o acúmulo de matéria orgânica. De forma contrária,

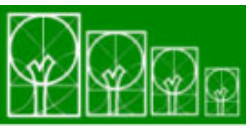

$\mathbf{S} \cdot \mathbf{B} \cdot \mathbf{A} \cdot \mathbf{U}$ Soc. Bras. de Arborização Urbana 
espécies de casca mais lisa como as palmeiras serviram como forófitos apenas para quatro plantas. Dentre as epífitas do centro, destacam-se aquelas da família Araceae, que são comumente usadas em projetos paisagísticos, e a espécie de árvore Ficus nitida, que pode crescer também como uma epífita. As hemiparasitas de ramos foram representadas pelas ervas-de-passarinho (Struthanthus vulgaris Eichler in Martius), presentes em 7,5\% das árvores. As ervas-de-passarinho causam danos sérios às árvores apenas em grandes infestações, e por isso não sugerimos o seu extermínio nas cidades, ao contrário das recomendações usuais (LEAL et al., 2006 e ROTTA, 2004). As ervas-de-passarinho contribuem para a alimentação das aves, e devem ser mantidas na arborização como fonte de alimento para avifauna. Contudo, sugerimos o manejo dessas plantas para evitar grandes infestações que poderiam ser danosas para as árvores, assim como recomendamos que fossem retiradas das árvores jovens, para não comprometer seu desenvolvimento inicial.
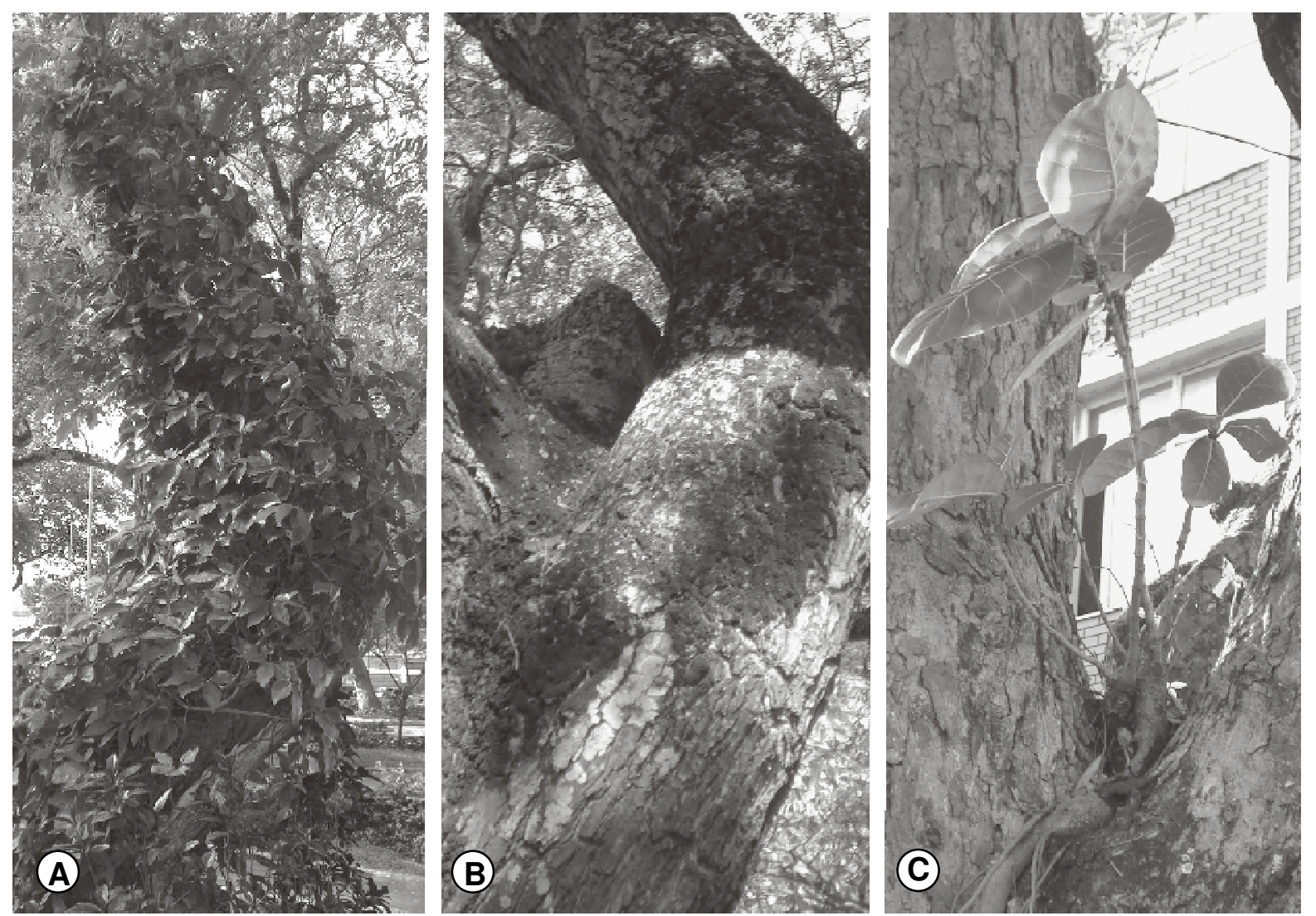

FIGURA 2. Organismos associados às árvores encontrados no bairro Centro. A) Araceae epífita. B) Briófitas. C) Moraceae epífita.

FIGURE 2. Organisms associated with the trees found in Aracaju's downtown. A) Araceae epiphyte. B) Bryophytes. C) Moraceae epiphyte. 
Através do exame da superfície externa do tronco e de áreas internas à casca solta, observamos cupins em $11,5 \%$ das árvores do bairro centro. No entanto, o número de árvores infestadas pode ser bem maior, uma vez que não foi possível realizar exames do interior das árvores, e alguns gêneros de cupins atacam somente o cerne e a árvore não demonstra sinais externos de infestação. A depender da intensidade do ataque, os cupins podem danificar a árvore seriamente e até mesmo levá-la a morte, já que se alimentam da parede celular encontrada na madeira e folhas (RUPPERT et al., 2005)

Outros organismos presentes nas árvores do centro são os lagartos, pássaros, fungos, abelhas, vespas, aranhas e outros artrópodes.

A espécie de árvore que sustenta a maior quantidade de tipos de organismos no Centro é a $P$. dulce. As características de sua casca, a presença de espinhos no ramos (que abrigam os animais e os protegem de predadores urbanos como gatos domésticos) e a elevada produção de flores e frutos durante o ano, além de sua abundância no local, fazem com que esta espécie seja a mais utilizada pelos organismos. Contudo, $P$. dulce é uma espécie exótica, e ainda não sabemos até que ponto ela está envolvida em interações com organismos nativos.

Alguns estudos têm apontado a dependência da fauna local pelas espécies vegetais introduzidas (SHAPIRO, 2002), e desta forma é necessária cautela antes da completa substituição das árvores exóticas por plantas locais. Por isso, sugerimos que essa substituição seja realizada de modo gradual, a medida que as árvores exóticas morram ou entrem em condições fitosanitárias que eliminem suas funções no ambiente urbano.

\section{CONCLUSÃO}

O bairro Centro da cidade de Aracaju-SE apresenta um número bastante significativo de indivíduos arbóreos, no entanto, pertencente a um pequeno número de espécies, o que caracteriza uma arborização homogênea. A maioria das espécies vegetais encontradas é considerada exótica, em relação à origem geográfica, causando impactos ainda não aferidos nas espécies animais e vegetais locais. Considerando que uma espécie arbórea exótica apresentou a maior quantidade de organismos associados, sugere-se que trabalhos avaliando a dependência das espécies animais do local a estas árvores exóticas sejam realizados para que assim seja elaborado um planejamento de quais espécies vegetais

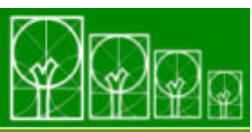

$\mathbf{S} \cdot \mathbf{B} \cdot \mathbf{A} \cdot \mathbf{U}$ Soc. Bras. de Arborização Urbana 
nativas poderiam substituí-las com menor prejuízo a estes animais, examinando atentamente qual a melhor maneira de organizar esta substituição.

\section{REFERÊNCIAS BIBLIOGRÁFICAS}

ALMEIDA NETO, J.X.; SILVA, H.; DANTAS, I.C.; ALMEIDA, M.A.X.; LOPES, M.E.S. Levantamento Quantitativo e Qualitativo de plantas arbóreas na cidade de Barra de Santa Rosa - PB. Revista de Biologia e Ciências da Terra, João Pessoa, v. 5, n. 2, 2005.

APG III. Este artigo foi compilado por Bremer, B.; Bremer, K.; Chase, M. W.; Fay, M. F.; Reveal, J. L; Soltis, D. E.; Soltis, P. S. e Stevens, P. F., os quais são igualmente responsáveis e foram listados aqui em ordem alfabética; contribuições de Anderberg, A. A.; Moore, M. J.; Olmstead, R. G.; Rudall, P. J.; Sytsma, K. J.; Tank, D. C.; Wurdack, K.; Xiang, J. Q.-Y. e Zmarzty, S. (em ordem alfabética). An update of the Angiosperm Phylogeny Group classification for the orders and families of flowering plants: APG III. Botanical Journal of the Linnean Society, London, v. 161, n. 2, 2009.

ARAÚJO, H. M. Elementos componentes do sistema ambiental físico de Aracaju. IN: ARAUJO, H. M.; VILAR, J. W. C.; WANDERLEY, L. L.; SOUZA, R. M. (Orgs.). O ambiente urbano: visões geográficas de Aracaju. Editora UFS, Aracaju, p. 15-43, 2006.

AUER, C.G.; GOMES, N.S.B.; GRIGOLETTI - JÚNIOR, A. A Armilariose em Pínus no Brasil. Comunicado técnico 117. Colombo: Embrapa, 2004.

CAMPOS-FARINHA, A.E.C., O.C. BUENO, M.C.G. CAMPOS; L.M. KATO. As formigas urbanas no Brasil: Retrospecto. Arquivos do Instituto Biológico, São Paulo, v. 64, p. 129133, 2002.

CNIP - CENTRO NORDESTINO DE INFORMAÇÕES SOBRE PLANTAS. Checklist das Plantas do Nordeste Brasileiro: Angiospermae e Gymnospermae. Disponível em: <http://www.cnip.org.br/bdpn/checklistNE.pdf>. Acessado em: 04 dez 2009.

COSTA, S.B.; PELLI, A.; G.P. CARVALHO, A.G. OLIVEIRA, P.R. SILVA, M.M. TEIXEIRA, E. MARTINS, A.P.S. TERRA, E.M. RESENDE, C.C.H.B. OLIVEIRA; C.A. MORAIS. Formigas como vetores mecânicos de microorganismos no Hospital Escola da Universidade Federal do Triângulo Mineiro. Revista da Sociedade Brasileira de Medicina Tropical, Uberaba. v. 39, p. 527-529, 2006.

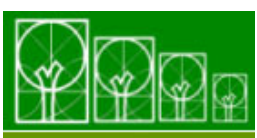

$\mathbf{S} \cdot \mathbf{B} \cdot \mathbf{A} \cdot \mathbf{U}$ Soc. Bras. de Arborização Urbana 
DANTAS, I. C.; SOUZA, C.M.C. Arborização urbana na cidade de Campina Grande - PB: Inventário e suas espécies. Revista de Biologia e Ciências da Terra, João Pessoa, v. 4, n. 2, 2004.

DREISTADT, S. H.; DAHLSTEN, D. L.; FRANKIE, G. W. Urban Forests and Insect Ecology. BioScience, Berkeley, v. 40, n. 3, p. 192-198, 1990.

FERREIRA, F.A.; MILANI, D. Avaliação de resistência de clones de eucalipto às infecções naturais de Cryphonectria cubensis, com nova metodologia. Revista Árvore, Viçosa, v. 28, n. 2, 2004.

GOOGLE MAPS. Disponível em: maps.google.com.br. Acessado em: 04 dez 2009.

HOLMGREN, P. K.; HOLMGREN, N. H. Index Herbariorum: A global directory of public herbaria and associated staff. New York Botanical Garden's Virtual Herbarium. Nova lorque, 1998 [continuamente atualizado] Disponível em: <http://sweetgum.nybg.org/ih/>. Acesso em 05 janeiro 2008.

IBGE.

Cidades.

Disponível

em: <http://www.ibge.gov.br/cidadesat/xtras/perfil.php?codmun=280030\&r=1> Acessado em: 20 de dezembro de 2009.

LEAL, L.; BUJOKAS, W.M.; BIONDI, D. Análise da infestação de erva-de-passarinho na arborização de ruas de Curitiba, PR. FLORESTA, Curitiba, v. 36, n. 3, 2006.

LIRA-FILHO, J. A.; PAIVA, H. N.; GONÇALVES, W. Paisagismo: princípios básicos. Viçosa: Aprenda Fácil, 162 p., 2001.

LORENZI, H.; SOUZA, H. M.; MEDEIROS-COSTA, J. T.; CERQUEIRA, L. S. C.; BEHR, N. Palmeiras do Brasil: nativas e exóticas. Nova Odessa: Editora Plantarum, 1996.

LORENZI, H. SOUZA, H. N.; TORRES, M. A. V.; BACHER, L. B. Árvores exóticas no Brasil. Nova Odessa: Editora Plantarum, 368 p., 2003.

MAGALHÃES, L.M.S. Arborização e florestas urbanas - terminologia adotada para a cobertura arbórea das cidades brasileiras. Série Técnica Floresta e Ambiente, Seropédica, v. 1 , p. 23-26, 2006.

MCKINNEY, M.L. Urbanization, Biodiversity, and Conservation. BioScience, Berkeley, v. 52, n. 10, p. 883-890, 2002.

MENESES, C.H.S.G.; SOUSA, E.B.M.; MEDEIROS, F.P.M.; ALBUQUERQUE, H.N.; SANTOS, L. Análise da arborização dos bairros do Mirante e Vila Cabral na cidade de (1) POP

$\mathbf{S} \cdot \mathbf{B} \cdot \mathbf{A} \cdot \mathbf{U}$ Soc. Bras. de Arborização Urbana

REVSBAU, Piracicaba - SP, v.5, n.4, p.22-39, 2010 
Campina Grande - PB. Revista de Biologia e Ciências da Terra, João Pessoa, v. 3, n. 2, 2003.

MURPHY, D.D. Desafios à diversidade biológica em áreas urbanas. In: WILSON, E.O. Biodiversidade. Rio de Janeiro: Nova Fronteira, 657 p., 1997.

NETO, E.M.L.; RESENDE, W.X.; SOUZA, R.M. Áreas verdes do Centro de Aracaju - SE : Análise fitogeográfica. Revista da Fapese, Aracaju, v.3, n. 2, p. 5-16, 2007.

OKE, T.R.; CROWTHER, J.M.; MCNAUGHTON, K.G.; MONTEITH, J. L.; GARDINER, B. The Micrometeorology of the Urban Forest [and Discussion]. Philosophical Transactions of the Royal Society of London. Series B, Biological Sciences, London, v. 324, n. 1223, p. 335-349, 1989.

PACHECO, R.; VASCONCELOS, H.L. Invertebrate conservation in urban areas: Ants in the Brazilian Cerrado. Landscape and Urban Planning, v. 81, p. 193-199, 2007.

PICKETT, S. T. A.; CADENASSO, M. L.; GROVE, J. M.; NILON, C. H.; POUYAT, R. V.; ZIPPERER, W. C.; CONSTAZA, R. Urban Ecological Systems: Linking Terrestrial Ecological, Physical, and Socioeconomic Components of Metropolitan Areas. Annual Review of Ecology and Systematics, v. 32, p. 127-157, 2007.

PIELOU, E. C. Species diversity and pattern diversity in the study of ecological succession. Journal of Theoretical Biology, v. 10, n. 2, 370-383, 1966.

PIRES, N. A. M. T.; MELO, M. S.; OLIVEIRA, D. E.; XAVIER-SANTOS, S. Diagnóstico da Arborização Urbana do Município de Goiandira, Goiás. Revista Brasileira de Biociências, Porto Alegre, v. 5, supl. 1, p. 537-539, 2007.

PIVETTA, K. F. L.; SILVA-FILHO, D. F. Arborização urbana. Boletim Acadêmico: Série arborização urbana, Jaboticabal, 2002.

ROCHA, R.T.; LELES, P.S.S.; NETO, S.N.O. Arborização de vias públicas em Nova Iguaçu, RJ: O Caso dos bairros Rancho Novo e Centro. Revista Árvore, Viçosa, v. 28, n. 4, p. 599607, 2004.

ROSSATO, D. R.; TSUBOY, M. S. F.; FREI, F. Arborização urbana na cidade de Assis-SP: uma abordagem quantitativa. Revista da Sociedade Brasileira de Arborização Urbana, Piracicaba, v. 3, n. 3, p. 1-16, 2008.

ROTTA, E. Autotrofia em Tripodanthus acutifolius (Ruiz \& Pav.) Thieg. (erva-de-passarinho) - um registro. Comunicado técnico, n. 115. Colombo: Embrapa, 2004. 
RUPPERT, E. E.; FOX, R.S.; BARNES, R.D. Zoologia dos invertebrados: uma abordagem funcional-evolutiva. $7^{a}$ ed. São Paulo: Roca, 2005.

SANTOS, W.R.A.; VARGAS, M.A.M. Apropriações na Construção do Urbano na Cidade de Aracaju/SE. Scientia Plena, Aracaju, v. 3, n. 5, p. 117-123, 2007.

SOARES, N.S.; ALMEIDA, L.O; GONÇALVES, C.A.; MARCOLINO, M.T.; BONETTI, A.M. Levantamento da diversidade de formigas (Hymenoptera: Formicidae) na região urbana de Uberlândia, MG. Neotropical entomology, Piracicaba, v. 35, n. 3, p. 324-328, 2006.

TANGLEY, L. The Urban Ecologist. BioScience, Berkeley, v. 36, n. 2, p. 68-71, 1986.

TRATALOS, J.; FULlER, R. A.; WARREN, P. H.; DAVIES, R. G.; GASTON, K. J. Urban form, biodiversity potential and ecosystem services. Landscape and Urban Planning, v. 83, p. 308-317, 2007.

TROPICOS. Botanical information system at the Missouri Botanical Garden. Disponível em: <http://www.tropicos.org> Acessado em: 15 de abril de 2010.

VILAR, J. W. C. Evolução da paisagem urbana do centro de Aracaju. In: ARAUJO, H. M.; VILAR, J. W. C.; WANDERLEY, L. L.; SOUZA, R. M. (Orgs.). O ambiente urbano: visões geográficas de Aracaju. Editora UFS, Aracaju, p. 49-71, 2006.

WORLD CHECKLIST OF SELECTED PLANT FAMILIES. The Board of Trustees of the Royal Botanic Gardens, Kew. Disponível em: <http://apps.kew.org/wcsp/home.do> Acessado em: 15 de abril de 2010.

ZIPPERER, W. C.; WU, J.; POUYAT, R. V. \& PICKETT, S. T. A. The Application of Ecological Principles to Urban and Urbanizing Landscapes. Ecological Applications, v. 10, n. 3, p. 685-688, 2000. 